\title{
Synthesis of the Antimalarial Drug FR900098 Utilizing the Nitroso- Ene Reaction
}

\author{
Andrey A. Fokin, Alexander G. Yurchenko, Vladimir N. Rodionov, Pavel A. Gunchenko, Raisa I. \\ Yurchenko, Armin Reichenberg, Jochen Wiesner, Martin Hintz, Hassan Jomaa, and Peter R. \\ Schreiner
}

\section{SUPPLEMENTARY INFORMATION}

\section{Experimental}

Nitroso-ene reaction of diethyl allylphosphonate (9). A solution of $1.8 \mathrm{~g}$ (10 mmol) of commercial (Aldrich) diethyl allylphosphonate (9) and $3.2 \mathrm{~g}(11.4 \mathrm{mmol})$ of the complex of nitrosocarbonyl methane with 9,10-dimethylanthracene ${ }^{34}$ in $70 \mathrm{~mL}$ of toluene was heated at $90{ }^{\circ} \mathrm{C}$ in a sealed glass tube for $16 \mathrm{~h}$. The reaction mixture was cooled and precipitated 9,10dimethylanthracene $(2.25 \mathrm{~g}, 11 \mathrm{mmol})$ was filtered off. The filtrate was concentrated under reduced pressure and the reaction mixture was separated by column chromatography on silica to give $25 \mathrm{mg}$ of 9,10-dimethylanthracene (hexane:THF =9:1) and $0.3 \mathrm{~g}(1.7 \mathrm{mmol})$ of starting compound 9 (ether). After changing the eluent for ether:methanol=95:5 0.37 g (1.5 mmol, 15\%) of diethyl $E$-[3-( $N$-acetyl- $N$-hydroxyamino)-propen-1-yl)]-phosphonate (11e) and, separately 0.30 g (1.2 mmol, 12\%) of diethyl Z-[3-( $N$-acetyl- $N$-hydroxyamino)-propen-1-yl)]-phosphonate (11z) as colorless liquids were isolated. Intermediate fractions contained $1.20 \mathrm{~g}(4.8 \mathrm{mmol}, 48 \%)$ of the mixture of $\mathbf{1 1 e}$ and $\mathbf{1 1 z}$ in an approximately 1:1 ratio.

Hydrogenation of diethyl [3-( $N$-acetyl- $N$-hydroxyamino)-propen-1-yl)]-phosphonate (11). A solution of $0.52 \mathrm{~g}(2 \mathrm{mmol})$ of the mixture of diastereomers of 11 in $50 \mathrm{~mL}$ of methanol was hydrogenated under 1 atm of $\mathrm{H}_{2}$ pressure at room temperature in presence of $4 \mathrm{mg}$ of $10 \%-\mathrm{Pd} / \mathrm{C}$. The reaction mixture was filtered and passed through a column with silica (hexane:ethylacetate 1:1) to give $0.5 \mathrm{~g}(1.9 \mathrm{mmol}, 95 \%)$ of $\mathbf{1 2}$.

Hydrolysis of diethyl [3-(N-acetyl- $N$-hydroxyamino)-propyl]-phosphonate (12) and preparation of FR900098 (1). To a solution of $253 \mathrm{mg}(1 \mathrm{mmol})$ of 12 in $0.4 \mathrm{~mL}$ of $\mathrm{CH}_{2} \mathrm{Cl}_{2} 1.2$ $\mathrm{g}(7.9 \mathrm{mmol})$ of trimethyl bromosilane was added at $0{ }^{\circ} \mathrm{C}$. The reaction mixture was stirred at room temperature for $4 \mathrm{~h}$ and concentrated in vacuo at room temperature. Water $(8 \mathrm{~mL})$ was added to the residue and the mixture was stirred overnight at room temperature, filtered and 
concentrated in vacuo to give $177 \mathrm{mg}(0.9 \mathrm{mmol}, 90 \%)$ of crude acid (13), which was rapidly neutralized with a solution of $41 \mathrm{mg}(1 \mathrm{mmol})$ of $\mathrm{NaOH}$ in $2 \mathrm{~mL}$ of deionized water. The mixture was stirred overnight, concentrated in vacuo to give $210 \mathrm{mg}$ of slightly yellowish oil, which was dissolved in $15 \mathrm{~mL}$ of methanol. Addition of acetone causes sedimentation of a white precipitate, which gave after filtration, washing with acetone and drying in vacuum at $60{ }^{\circ} \mathrm{C} 197$ mg (0.9 mmol, 90\%) of FR900098 (1) as a white powder, which was identical to the standard sample from the NMR data and the results of the HPLC analysis.

Preparation of dibenzyl allylphosphonate (14). To a stirred solution of $5.2 \mathrm{~g}(20 \mathrm{mmol})$ of dibenzyl phosphite in $40 \mathrm{~mL}$ of dry ether at $0{ }^{\circ} \mathrm{C} 13 \mathrm{~mL}(21 \mathrm{mmol})$ of a $1.6 \mathrm{M}$ solution of $n$-BuLi in $n$-hexane was added in portions at $0{ }^{\circ} \mathrm{C}$, followed by the dropwise addition of $7 \mathrm{~g}(58 \mathrm{mmol})$ of freshly distilled allylbromide. The reaction mixture was stirred at room temperature for $4 \mathrm{~h}$, cooled with an ice bath, and quenched with water. The organic layer was separated, washed with water, brine, dried over $\mathrm{Na}_{2} \mathrm{SO}_{4}$, evaporated, and filtered through silica gel to give $5.8 \mathrm{~g}(90 \%, 19$ mmol) of a slightly yellow liquid, whose spectral data were identical to those previously described. $^{43}$

Nitroso-ene reaction of dibenzyl allylphosphonate (14). A mixture of $1.5 \mathrm{~g}$ (5 mmol) of dibenzyl allylphosphonate (14) and $0.47 \mathrm{~g}(6.2 \mathrm{mmol})$ of $\mathbf{1 0}$ in $20 \mathrm{~mL}$ of toluene was heated at 90 ${ }^{\circ} \mathrm{C}$ as above for $36 \mathrm{~h}$. 9,10-dimethylanthracene $(0.93 \mathrm{~g}, 4.5 \mathrm{mmol})$ was filtered off and column chromatography gave (15 mg) of 9,10-dimethylanthracene, $0.2 \mathrm{~g}$ of $\mathbf{9}, 0.22 \mathrm{~g}(1.5 \mathrm{mmol}, 12 \%)$ of dibenzyl $E$-[3-(N-acetyl- $N$-hydroxyamino)-propen-1-yl)]-phosphonate (15e) and $0.19 \mathrm{~g}(0.5$ mmol, 10\%) of dibenzyl Z-[3-( $N$-acetyl- $N$-hydroxyamino)-propen-1-yl)]-phosphonate (15z). Intermediate fractions contained $0.56 \mathrm{~g}(1.5 \mathrm{mmol}, 30 \%)$ of $\mathbf{1 5 e}$ and $\mathbf{1 5 z}$ in an approximately $1: 1$ ratio.

Hydrogenation of benzyl [3-( $N$-acetyl- $N$-hydroxyamino)-propen-1-yl)]-phosphonate (15) and preparation of FR900098 (1). A solution of $0.5 \mathrm{~g}(1.3 \mathrm{mmol})$ of the mixture of diastereomers of $\mathbf{1 5}$ in $30 \mathrm{~mL}$ of methanol was hydrogenated under 1 atm of $\mathrm{H}_{2}$ pressure at room temperature in the presence of $3 \mathrm{mg}$ of $10 \%-\mathrm{Pd} / \mathrm{C}$ for $24 \mathrm{~h}$. The reaction mixture was filtered, evaporated, the residue was washed with ether and neutralized by $1.2 \mathrm{~mL}$ of a $1 \mathrm{M}$ solution of $\mathrm{NaOH}$ in water. The mixture was stirred for $2 \mathrm{~h}$ at room temperature and evaporated to give 0.26 $\mathrm{g}(95 \%, 1.2 \mathrm{mmol})$ of FR900098 (1), which was identical to the standard sample. 


\section{Spectral data}

Diethyl E-[3-(N-acetyl-N-hydroxyamino)-propen-1-yl)]-phosphonate (11e), colorless liquid. ${ }^{1} \mathrm{H}-\mathrm{NMR}\left(\mathrm{CDCl}_{3}, \delta, p p m\right): 1.30(\mathrm{t}, J=7 \mathrm{~Hz}, 6 \mathrm{H}), 2.17(\mathrm{~s}, 3 \mathrm{H}), 4.03(\mathrm{~m}, 4 \mathrm{H}), 4.40$ (bs, 2 $\mathrm{H}), 5.87(\mathrm{~m}, 1 \mathrm{H}), 6.71(\mathrm{~m}, 1 \mathrm{H}), 9.84(\mathrm{bs}, 1 \mathrm{H}) .{ }^{13} \mathrm{C}-\mathrm{NMR}\left(\mathrm{CDCl}_{3}, \delta, p p m\right): 16.3(\mathrm{~d}, J=6 \mathrm{~Hz})$, 20.3, $50.4(\mathrm{~d}, J=24 \mathrm{~Hz}), 62.3(\mathrm{~d}, J=5 \mathrm{~Hz}), 117.9(\mathrm{~d}, J=188 \mathrm{~Hz}), 147.7$ (d, $J=5 \mathrm{~Hz})$, 172.6. HR-MS: Found: 251.0921, cald. for $\mathrm{C}_{9} \mathrm{H}_{18} \mathrm{NO}_{5} \mathrm{P} 251.0923$.

Diethyl Z-[3-(N-acetyl-N-hydroxyamino)-propen-1-yl)]-phosphonate (11z), colorless liquid. ${ }^{1} \mathrm{H}-\mathrm{NMR}\left(\mathrm{CDCl}_{3}, \delta, p p m\right): 1.26(\mathrm{t}, J=7 \mathrm{~Hz}, 6 \mathrm{H}), 2.10(\mathrm{~s}, 3 \mathrm{H}), 3.98(\mathrm{~m}, 4 \mathrm{H}), 4.50(\mathrm{bs}, 2$ $\mathrm{H}), 5.66(\mathrm{~m}, 1 \mathrm{H}), 6.49(\mathrm{~m}, 1 \mathrm{H}), 9.88(\mathrm{bs}, 1 \mathrm{H}) .{ }^{13} \mathrm{C}-\mathrm{NMR}\left(\mathrm{CDCl}_{3}, \delta, p p m\right): 16.3(\mathrm{~d}, J=7 \mathrm{~Hz})$, 31.0, $49.2(\mathrm{~d}, J=9 \mathrm{~Hz}), 62.5(\mathrm{~d}, J=6 \mathrm{~Hz}), 116.6(\mathrm{~d}, J=184 \mathrm{~Hz}), 148.8,172.8$. HR-MS: Found 251.0918, cald. for $\mathrm{C}_{9} \mathrm{H}_{18} \mathrm{NO}_{5} \mathrm{P} 251.0923$.

Diethyl [3-(N-acetyl-N-hydroxyamino)-propyl]-phosphonate (12), colorless liquid. ${ }^{1} \mathrm{H}$ NMR $\left(\mathrm{CDCl}_{3}, \delta, p p m\right): 1.26(\mathrm{t}, J=7 \mathrm{~Hz}, 6 \mathrm{H}), 1.66-1.99(\mathrm{~m}, 4 \mathrm{H}), 2.09(\mathrm{~s}, 3 \mathrm{H}), 3.66(\mathrm{t}, J=6$ $\mathrm{Hz}, 2 \mathrm{H}), 4.00$ (qt, $4 \mathrm{H}), 9.71(\mathrm{bs}, 1 \mathrm{H}) .{ }^{13} \mathrm{C}-\mathrm{NMR}\left(\mathrm{CDCl}_{3}, \delta, p p m\right): 16.4(\mathrm{~d}, J=6 \mathrm{~Hz}), 19.3(\mathrm{~d}, J$ $=5 \mathrm{~Hz}), 20.6(\mathrm{~d}, J=18 \mathrm{~Hz}), 23.6,47.5(\mathrm{~d}, J=11 \mathrm{~Hz}), 62.1(\mathrm{~d}, J=6 \mathrm{~Hz}), 172.6$. HR-MS:

Found: 251.0921, cald. for $\mathrm{C}_{9} \mathrm{H}_{18} \mathrm{NO}_{5} \mathrm{P}$ 251.0923. HR-MS: Found 253.1062, cald. for $\mathrm{C}_{9} \mathrm{H}_{20} \mathrm{NO}_{5} \mathrm{P}$ 251.1079.

Dibenzyl E-[3-(N-acetyl-N-hydroxyamino)-propen-1-yl)]-phosphonate (15e), colorless liquid. ${ }^{1} \mathrm{H}-\mathrm{NMR}\left(\mathrm{CDCl}_{3}, \delta, p p m\right): 2.04(\mathrm{~s}, 3 \mathrm{H}), 4.76(\mathrm{bd}, 2 \mathrm{H}), 5.00(\mathrm{~m}, 4 \mathrm{H}), 5.78(\mathrm{~m}, 1 \mathrm{H})$, $6.50(\mathrm{~m}, 1 \mathrm{H}), 7.34(\mathrm{~m}, 10 \mathrm{H}), 9.23(\mathrm{bs}, 1 \mathrm{H}) .{ }^{13} \mathrm{C}-\mathrm{NMR}\left(\mathrm{CDCl}_{3}, \delta, p p m\right): 19.7,29.8,67.4(\mathrm{~d}, J=$ $6 \mathrm{~Hz}), 119.8$ (d, $J=185 \mathrm{~Hz}), 128.0,128.6,128.7,135.9,146.4,169.2$. HR-MS: Found: 375.1171 , cald. for $\mathrm{C}_{19} \mathrm{H}_{22} \mathrm{NO}_{5} \mathrm{P} 375.1136$.

Dibenzyl Z-[3-(N-acetyl-N-hydroxyamino)-propen-1-yl)]-phosphonate (15z), colorless liquid. ${ }^{1} \mathrm{H}-\mathrm{NMR}\left(\mathrm{CDCl}_{3}, \delta, p p m\right): 2.00(\mathrm{~s}, 3 \mathrm{H}), 4.39(\mathrm{bs}, 2 \mathrm{H}), 5.01(\mathrm{~m}, 4 \mathrm{H}), 5.87(\mathrm{t}, J=18 \mathrm{~Hz}$, $1 \mathrm{H}), 6.64(\mathrm{~m}, 1 \mathrm{H}), 7.34(\mathrm{~m}, 10 \mathrm{H}), 9.89$ (bs, $1 \mathrm{H}) .{ }^{13} \mathrm{C}-\mathrm{NMR}\left(\mathrm{CDCl}_{3}, \delta, p p m\right): 20.0,29.7,67.5$ $(\mathrm{d}, J=6 \mathrm{~Hz}), 119.8(\mathrm{~d}, J=190 \mathrm{~Hz}), 128.0,128.5,128.6,136.0(\mathrm{~d}, J=7 \mathrm{~Hz}), 145.1,168.4$. HRMS: Found: 375.1171, cald. for $\mathrm{C}_{19} \mathrm{H}_{22} \mathrm{NO}_{5} \mathrm{P} 375.1236$. 


\section{XYZ-Coordinates of computed species}

\begin{tabular}{|c|}
\hline S1, RB3PW91/6-? \\
\hline $\mathrm{HF}=-1125.6401191$ \\
\hline C 0.356992712 .997240221 .09005952 \\
\hline N 1.640025861 .793364670 .35168426 \\
\hline O $1.188845970 .68710034-0.05341499$ \\
\hline H -0.30598086 0.377822890 .85097447 \\
\hline C - 1.047138571 .014522971 .44727915 \\
\hline 5589940.89064163 \\
\hline C 2.788159971 .688454221 .25754874 \\
\hline C 3.749408130 .598901970 .87144297 \\
\hline O 2.983942842 .505236282 .13196041 \\
\hline P -2.714828460.30903944 1.2 \\
\hline 197000 \\
\hline $\mathrm{H} 0$ \\
\hline 18748610.55206287 \\
\hline Н $4.063449630 .71527282-0.17011374$ \\
\hline Н 4.614257340 .640598261 .53303891 \\
\hline Н $3.24566364-0.369973700 .94335477$ \\
\hline 04111 \\
\hline 5794321 \\
\hline 393562.47939317 \\
\hline O -3.78208056 0.965924512.08538974 \\
\hline O - $2.40407636-1.233809921 .67072330$ \\
\hline C $-3.47185296-2.085340882 .15220300$ \\
\hline 187762922.98 \\
\hline $2-2.59178424$ \\
\hline 77899472.73339720 \\
\hline H -4.00278740 -2.49354084 1.28419450 \\
\hline Н -3.64573175 -3.86344846 3.33928792 \\
\hline H -2.14112452 -3.77082641 2.40294383 \\
\hline Н -2.34439848 -2.774931373.85926984 \\
\hline H -2.40321455 -0.83496974 -3.35398651 \\
\hline H -3.97729720 - $0.73910986-2.53518890$ \\
\hline $077831120.74437212-2.90528786$ \\
\hline H - $1.266296220 .04364460-1.31323470$ \\
\hline H -2.17287689-1.43607248 -0.93229489 \\
\hline
\end{tabular}

TS2, RB3PW91/6-31+G**

$\mathrm{HF}=-1125.6407562$

C $1.97809491-0.030329501 .77258166$ N $0.62334854-0.620043742 .89556104$ O - $0.07492470-1.571102492 .46598541$ H $0.10889414-1.639052050 .71434912$ C $0.87264214-1.28343997-0.05652764$ C $2.05636942-0.958777070 .73669627$ C -0.163467510 .559008533 .35305989$ C -1.636569890 .337226333 .51554198$ O 0.441370641 .550329763 .68666722 P $0.000076520 .11193806-0.83692842$ H $2.89339108-1.652648350 .70047890$ H 1.417558190 .886184921 .58189926 H 2.830463580 .091824182 .43210963 H - $1.82910887-0.481452074 .21520710$ H - 2.086837351 .260034643 .88041424 H -2.06908515 0.048490972 .55383251 O $0.981617770 .75103981-1.94954685$ C $1.57557197-0.03582009-2.99842473$ O -0.492183061 .159123520 .09396403$ O $-1.13460112-0.69325614-1.66269851$ C $-2.33488743-0.00526071-2.09094554$ C $-3.38779990-1.04570403-2.40076153$ C $2.207969320 .91015983-3.99614133$ H -2.65529057 $0.67179955-1.29306192$ H - $2.095059150 .59379578-2.97727133$ H $-4.30272160-0.55280588-2.74564071$ H -3.04761897-1.72710125 -3.18614686 H -3.62714326 -1.63413151 - 1.51049636 H $2.686169410 .33864666-4.79836710$ H $1.454784451 .56759361-4.43922781$ H $2.966663961 .53134205-3.51225104$ H $2.33222101-0.69864050-2.55952742$ H $0.80345906-0.65442339-3.47119552$ H $1.03276240-2.08832945-0.77848767$ 


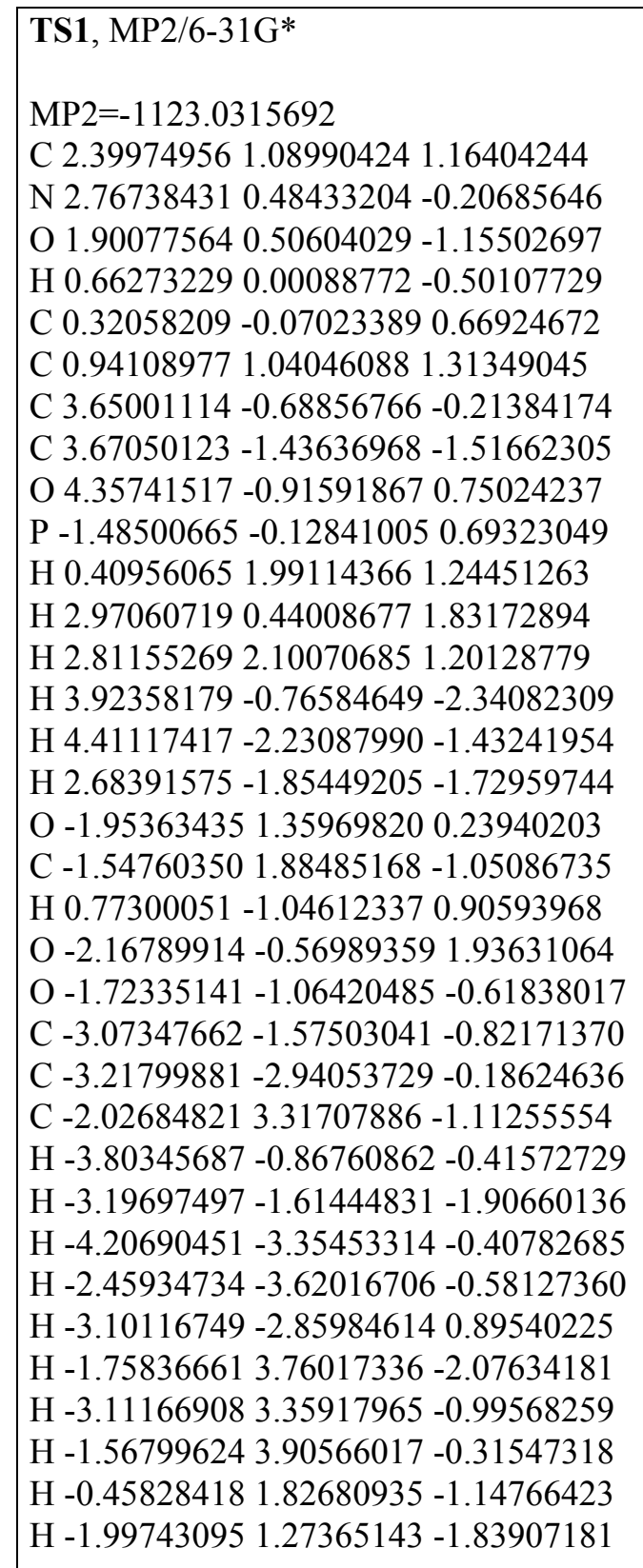

TS2, MP2/6-31G*

MP2 $=-1123.0377199$

C $1.94441676-1.812273400 .72873348$

N $2.57163294-0.434149260 .41271813$

O 2.314833550 .567450011 .20374096

H 0.972681560 .435432461 .53631660

C $0.08385973-0.467860501 .63477644$

C $0.87810136-1.643612611 .71182744$

C $2.71858439-0.10845693-1.00265364$

C $2.866737811 .35480068-1.30251720$

O $2.88053659-1.01726622-1.79489071$

P - $0.80389712-0.099178920 .11074792$

H $1.08699918-2.002062362 .72100288$

H $1.62631380-2.10609515-0.27508864$

H $2.73782386-2.480933981 .06687929$

H $3.713384821 .77585489-0.75494746$

H $3.024618951 .45566377-2.37601169$

H $1.965754571 .88781920-1.00039477$

O - $2.03365920-1.134152140 .19454350$

C -3.04089035 - $1.06478757-0.85712193$

H $-0.53781699-0.264054972 .50925938$

O $0.03292625-0.09438713-1.12847606$

O -1.569466331 .309897400 .39801884$

C -0.894075012 .537245120 .01357010$

C -1.838207973 .676755010 .31984444$

C -3.91948701 -2.28413475 -0.70616048

H 0.037352842 .627007000 .58432298

H -0.64626883 2.48588996-1.05011798

H - 1.375419154 .629495890 .04625747

H - $2.764663783 .56212992-0.24687522$

H -2.07922949 3.69702651 1.38457674

H $-4.70478537-2.27337241-1.46782545$

H -3.32772375 -3.19378335 -0.82454484

H $-4.38800610-2.295190820 .28004516$

H -3.60421320 - $0.13611791-0.73002277$

H -2.53694829 -1.04779442 -1.82798689 


\begin{tabular}{|c|c|}
\hline TS1, MP2/cc-pVDZ & TS2, MP2/cc-pVDZ \\
\hline MP2 $=-1123.2122118$ & MP2 $=-1123.2188824$ \\
\hline C 2.431920681 .099613431 .17943792 & C $1.95476983-1.835609140 .75339189$ \\
\hline N $2.758510160 .49745023-0.19889693$ & N $2.55286551-0.457822230 .40473484$ \\
\hline O $1.883501020 .53862276-1.11961090$ & O 2.316738660 .537548341 .18542141 \\
\hline Н $0.675445930 .03916656-0.47399677$ & H 1.009640750 .420770891 .53768622 \\
\hline C $0.34026530-0.066968670 .70001668$ & C $0.10186635-0.464686731 .66653203$ \\
\hline C 0.965232111 .034885471 .38130444 & C $0.88128458-1.664824431 .74688303$ \\
\hline C $3.63954589-0.69250614-0.22892309$ & C $2.67530981-0.14965222-1.02998564$ \\
\hline C $3.58429709-1.45248494-1.53024586$ & C $2.800284571 .31977402-1.33682100$ \\
\hline O $4.37842337-0.917884280 .70362511$ & O $2.83120607-1.06234928-1.80957874$ \\
\hline P - $1.47155110-0.141526840 .71743282$ & P - $0.79574722-0.084870060 .14383971$ \\
\hline Н 0.426955491 .992604611 .30179249 & H $1.12302553-1.982249552 .77234869$ \\
\hline H 3.037867540 .454225091 .83264079 & H $1.63900334-2.15568247-0.25165711$ \\
\hline Н 2.827214992 .125924761 .20373191 & Н $2.76204678-2.490670511 .11153611$ \\
\hline Н $3.78219876-0.78050134-2.37865587$ & Н $3.663144711 .75039690-0.80546403$ \\
\hline Н $4.33515849-2.25199818-1.48675492$ & Н $2.930052611 .42475884-2.42196513$ \\
\hline Н $2.57858506-1.87605119-1.67825617$ & H $1.894536351 .84438231-1.00679047$ \\
\hline O - 1.950063431 .364744740 .25611835 & O -2.05173112 -1.11870850 0.24029448 \\
\hline C - $1.489998341 .89041573-1.00740948$ & C -3.03013935 -1.03385893 -0.82782243 \\
\hline Н $0.80543888-1.055581040 .89847807$ & H -0.51191003 -0.22988088 2.54930476 \\
\hline O -2.18891190 -0.60006325 1.94845819 & O $0.02080432-0.07761662-1.12534495$ \\
\hline O - $1.68076139-1.07270311-0.62968672$ & O - 1.562792481 .343832500 .45242459 \\
\hline C -3.02774414 -1.56491504-0.84926956 & C -0.890094362 .556993660 .04823220 \\
\hline C $-3.21561876-2.92389468-0.19705961$ & C - 1.843977443 .707195360 .30630629 \\
\hline C -2.06978939 3.28488091 -1.14825522 & C $-3.86596705-2.29685873-0.77262888$ \\
\hline H -3.76575126 -0.83867689-0.46440072 & Н 0.040455192 .676960340 .63413916 \\
\hline H -3.14145208 -1.62182770 -1.94393253 & H -0.61862322 $2.48716885-1.01889126$ \\
\hline H -4.21575116 -3.32346936-0.43648978 & H - 1.373710604 .661446680 .01707622 \\
\hline H -2.45652467 -3.63091640 -0.56859572 & H -2.76662092 $3.57570494-0.28045131$ \\
\hline H -3.11880269-2.83012934 0.89487788 & H -2.111165583.75329380 1.37347880 \\
\hline H - $1.756907023 .72919444-2.10778073$ & H -4.64059838 -2.27046450 -1.55670911 \\
\hline H -3.16986797 $3.24673574-1.11662058$ & H -3.23082285 -3.18213225 -0.93023454 \\
\hline H -1.72012143 $3.93104781-0.32804177$ & H -4.35840895 -2.38746405 0.20810246 \\
\hline H -0.38566106 $1.91927339-1.02647587$ & H -3.64581560 -0.13140512-0.67029475 \\
\hline H - $1.829065981 .23086675-1.82541085$ & H -2.50710882 -0.94061821 -1.79554756 \\
\hline
\end{tabular}


4. The copies of NMR-spectra 


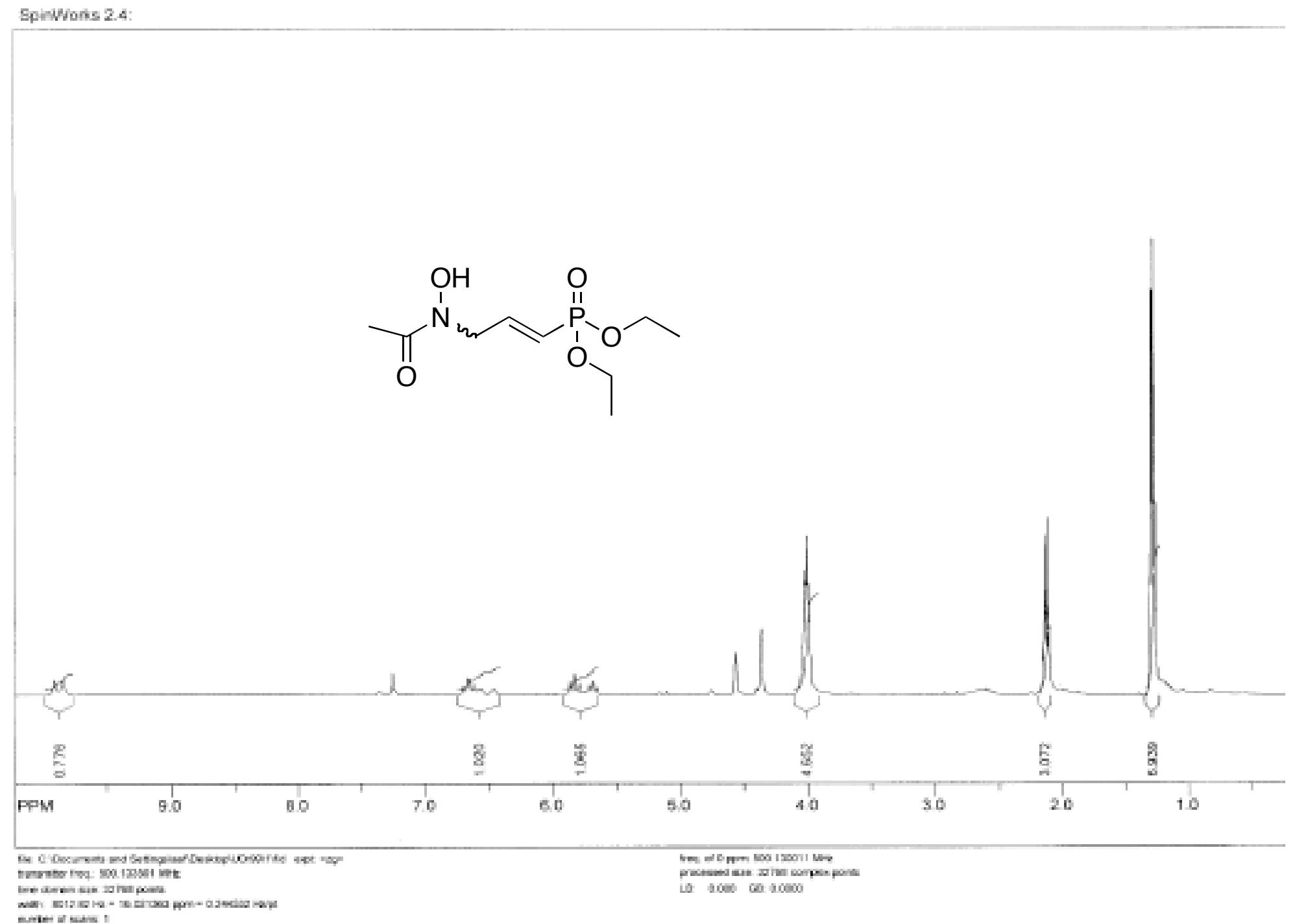

Figure S1. The ${ }^{1} \mathrm{H}$ NMR spectra of the compound $\mathbf{1 1}$. 


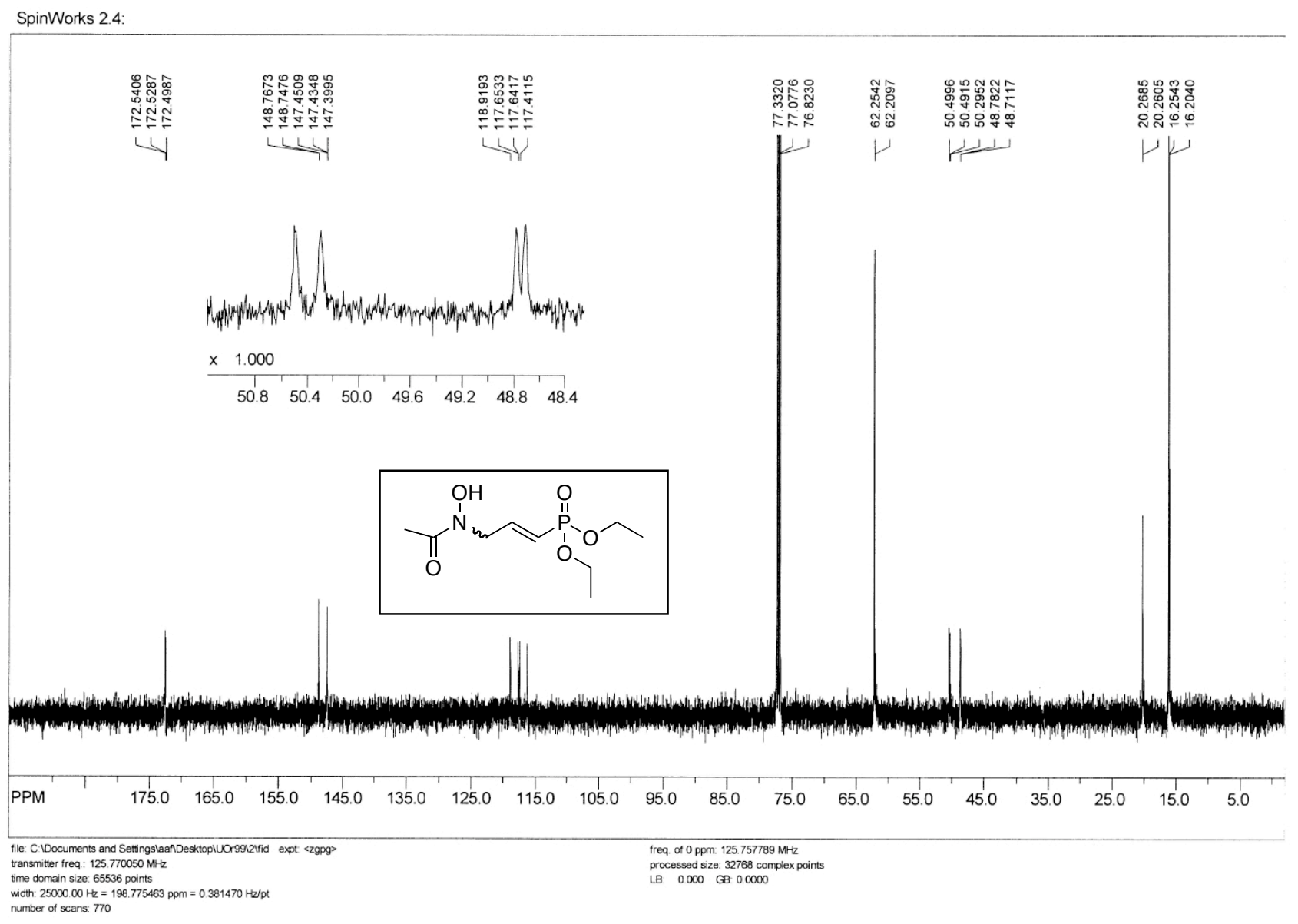

Figure S2. The ${ }^{13} \mathrm{C}$ NMR spectra of the compound $\mathbf{1 1}$. 


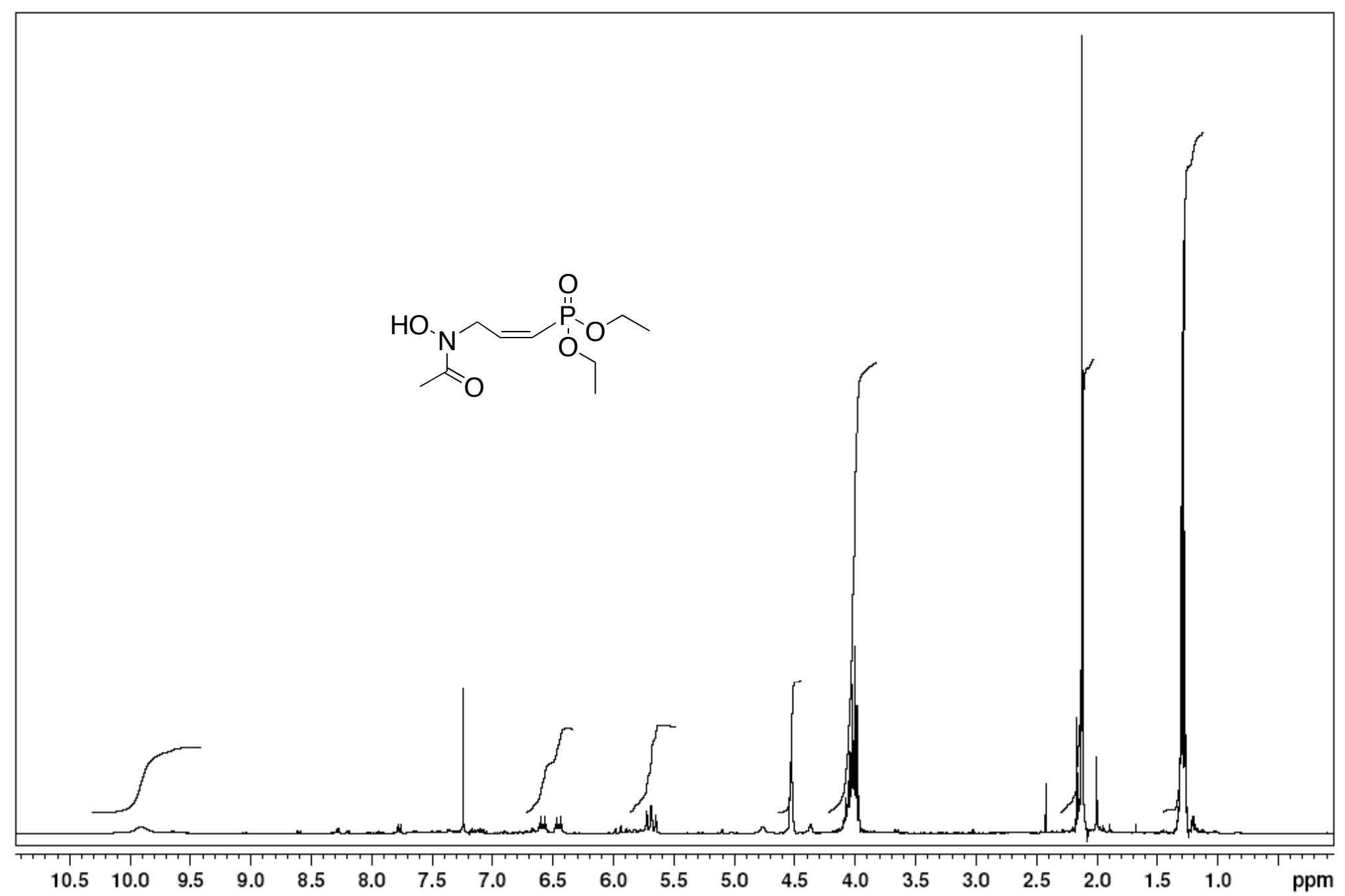

Figure S3. The ${ }^{1} \mathrm{H}$ NMR spectra of the compound $\mathbf{1 1 z}$. 


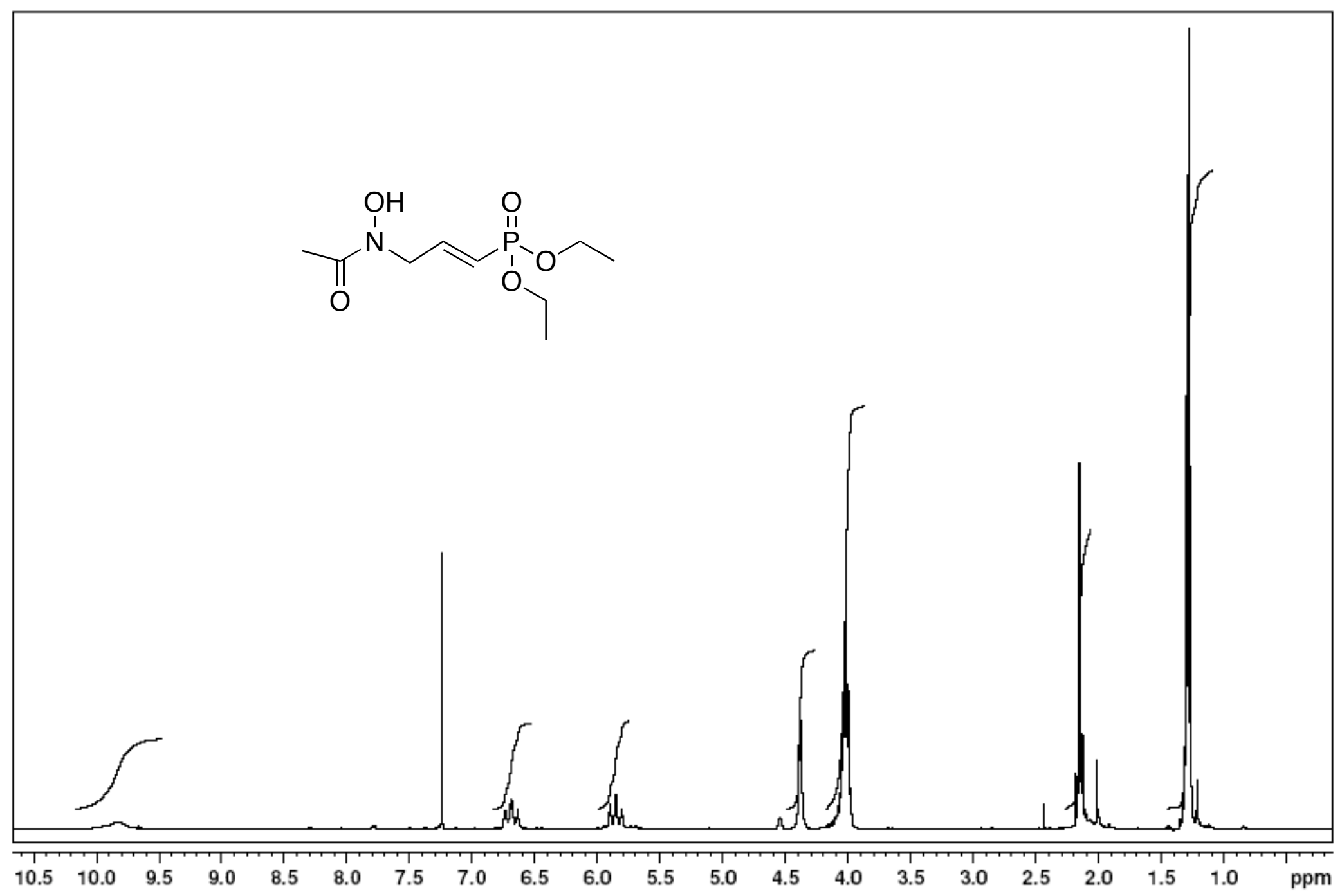

Figure S4. The ${ }^{1} \mathrm{H}$ NMR spectra of the compound 11e. 


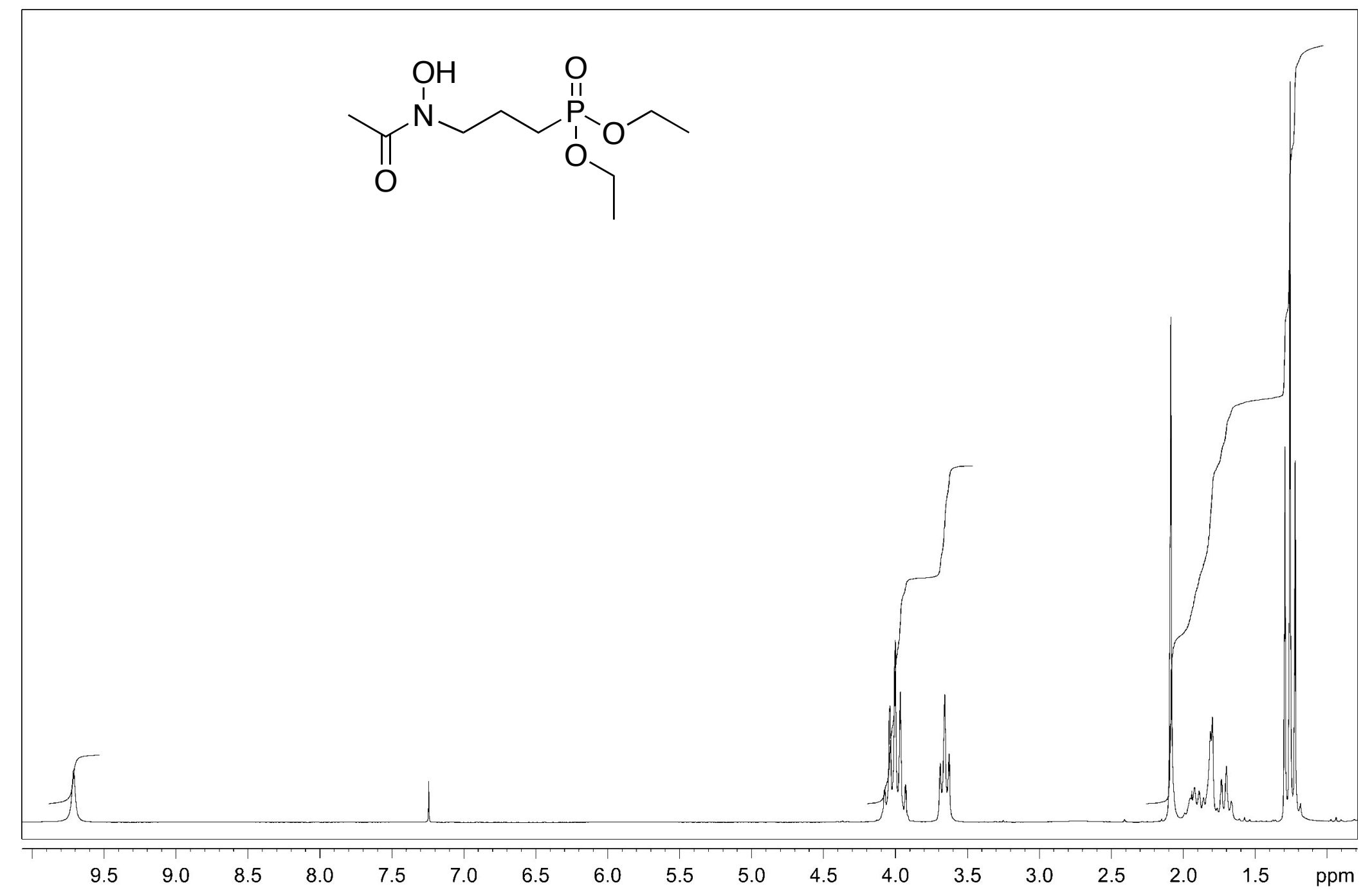

Figure S5. The ${ }^{1} \mathrm{H}$ NMR spectra of the compound $\mathbf{1 2}$. 


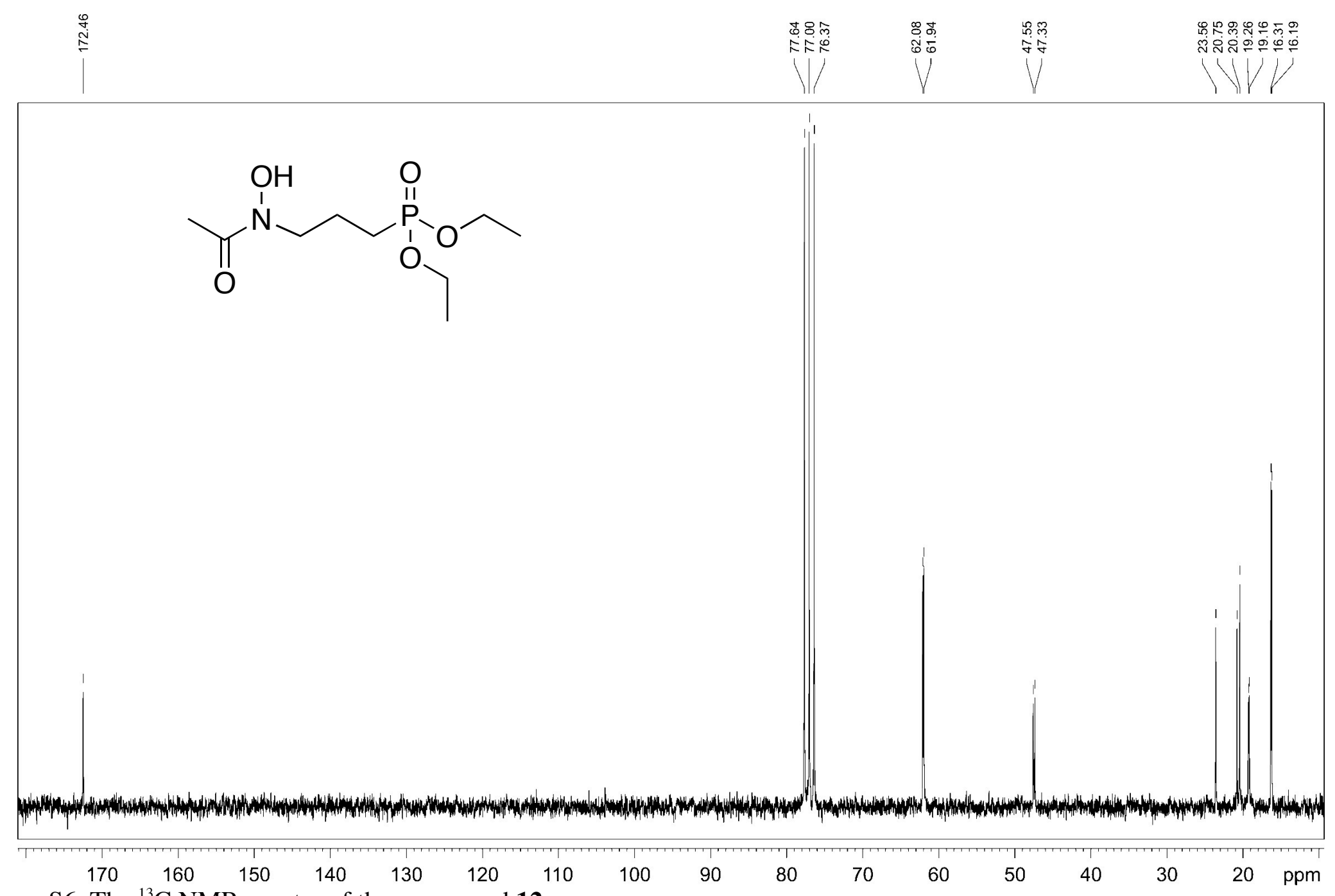

Figure S6. The ${ }^{13} \mathrm{C}$ NMR spectra of the compound 12. 


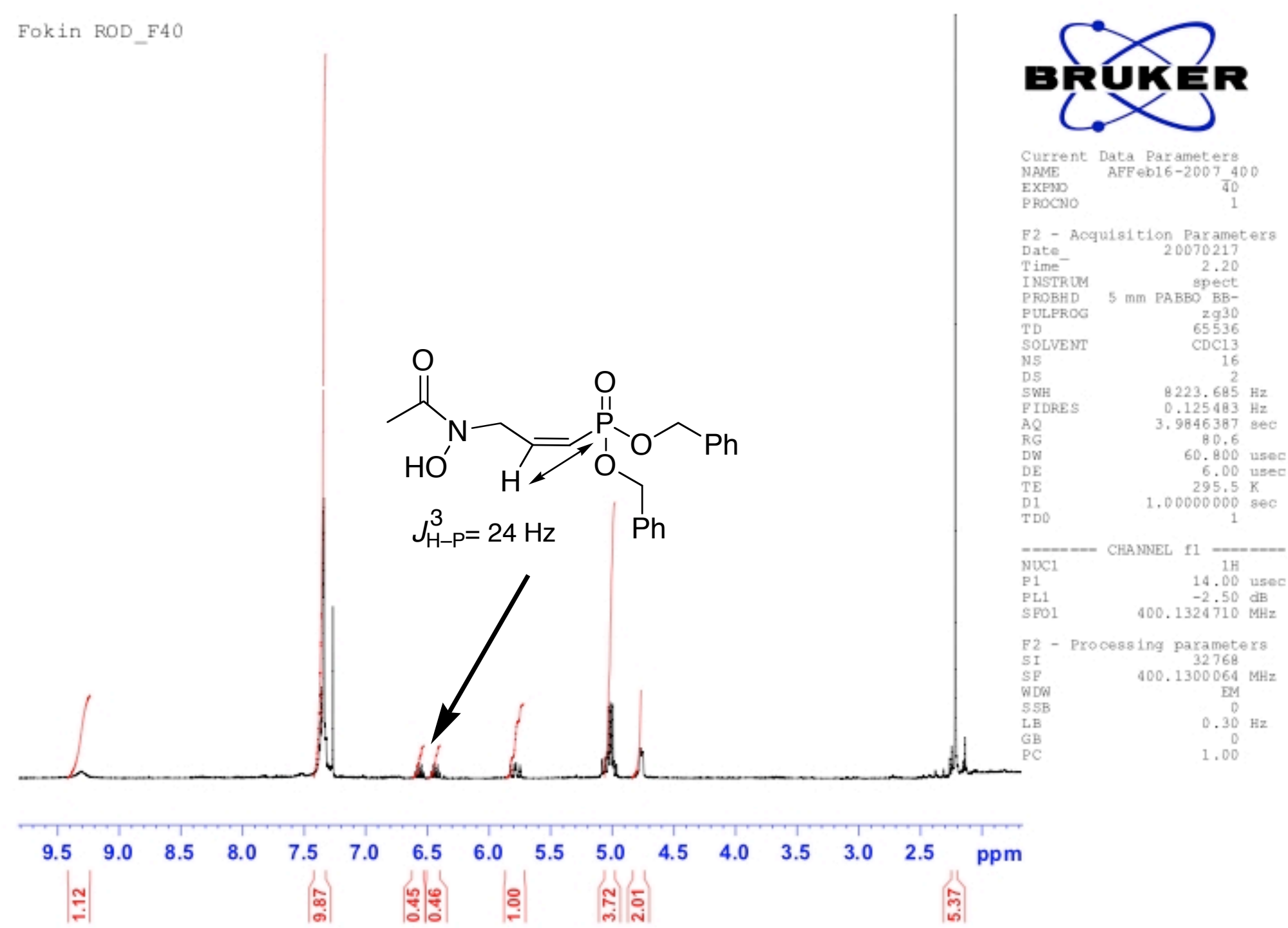

Figure S7. The ${ }^{13} \mathrm{H}$ NMR spectra of the compound $15 \mathbf{z}$. 


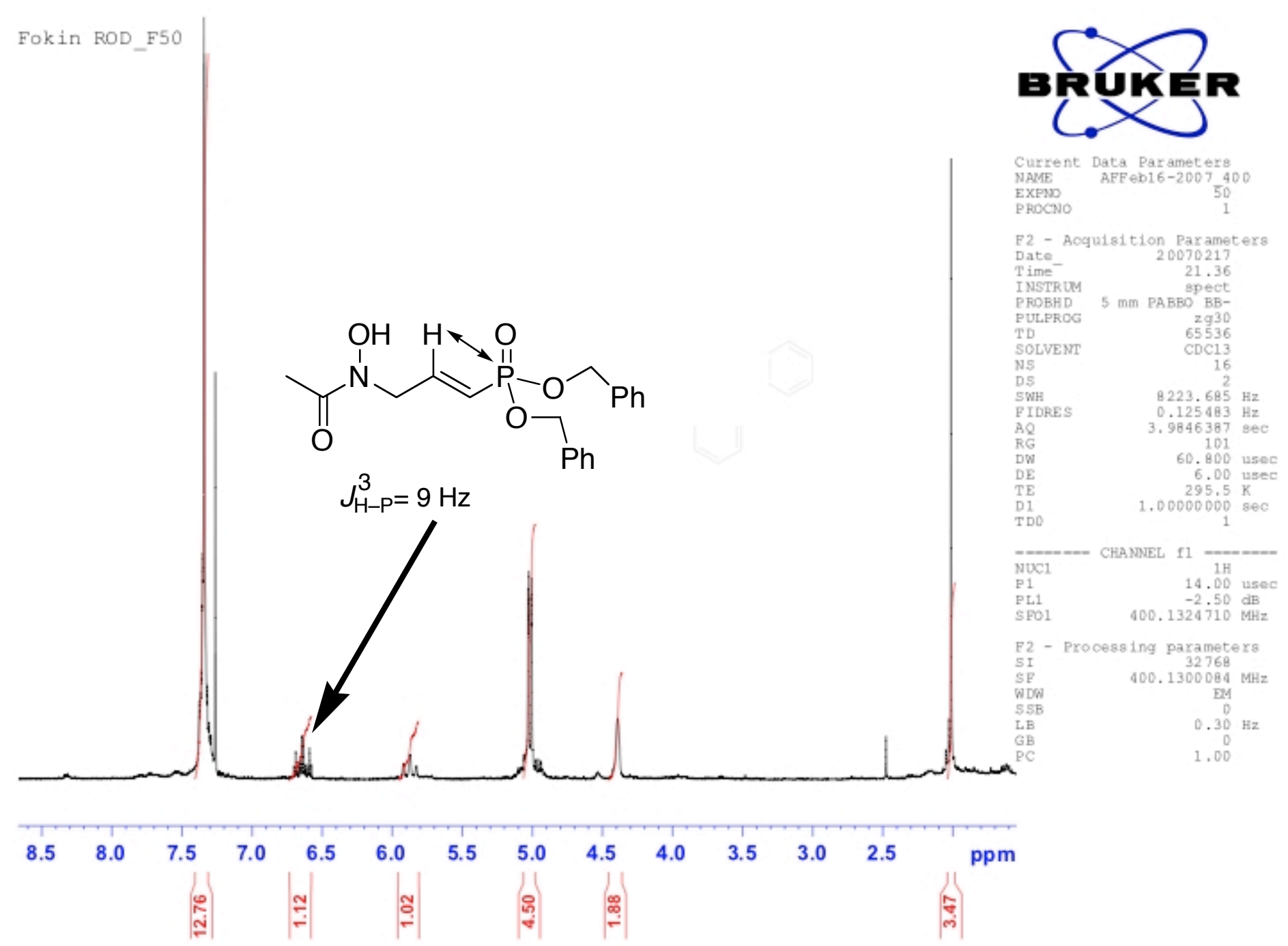

Figure S8. The ${ }^{13} \mathrm{H}$ NMR spectra of the compound $15 \mathbf{e}$. 


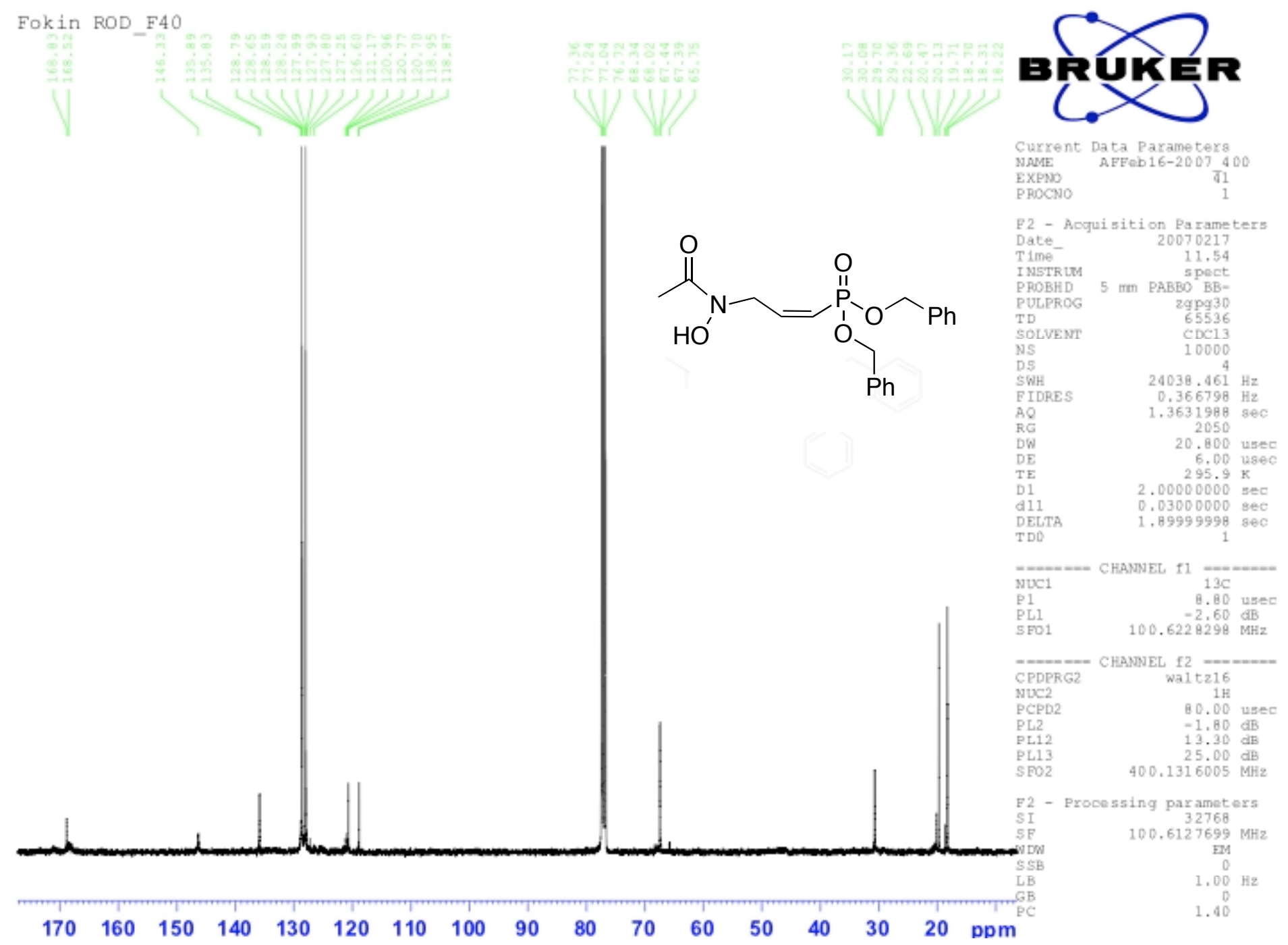

Figure S9. The ${ }^{13} \mathrm{C}$ NMR spectra of the compound $\mathbf{1 5 z}$. 


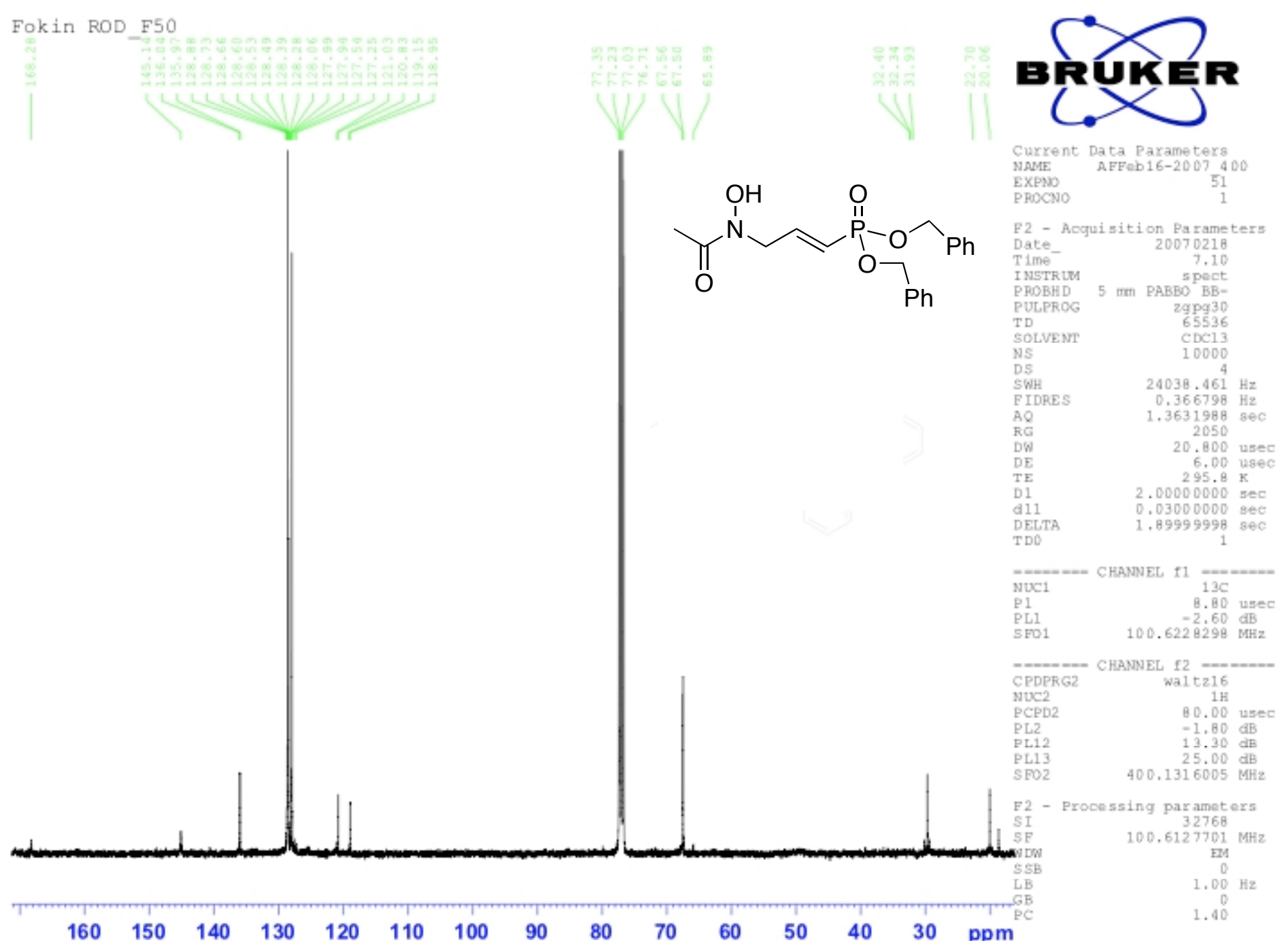

Figure S10. The ${ }^{13} \mathrm{C}$ NMR spectra of the compound $\mathbf{1 5 e}$. 


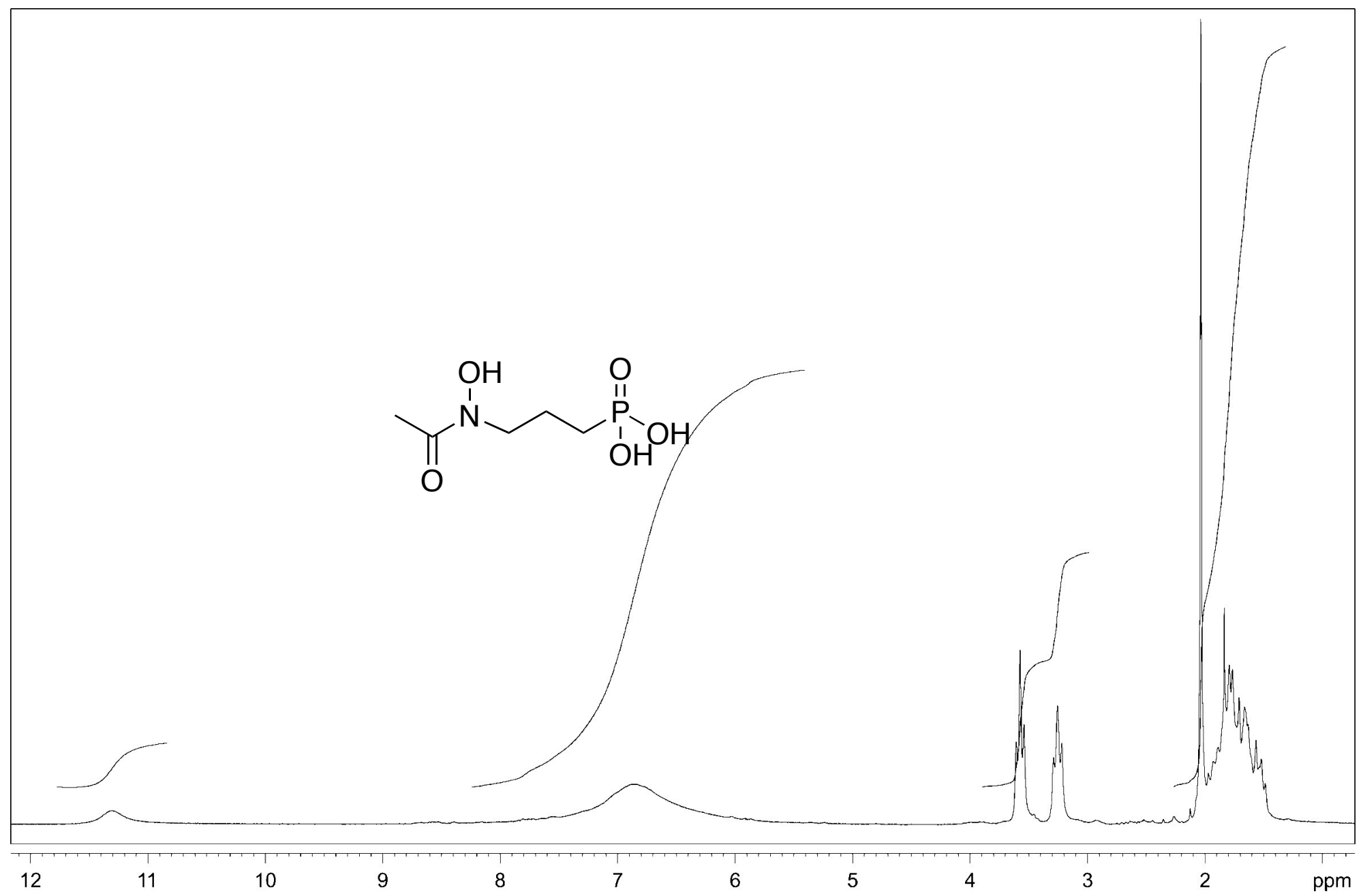

Figure S11. The ${ }^{1} \mathrm{H}$ NMR spectra of the compound 13. 


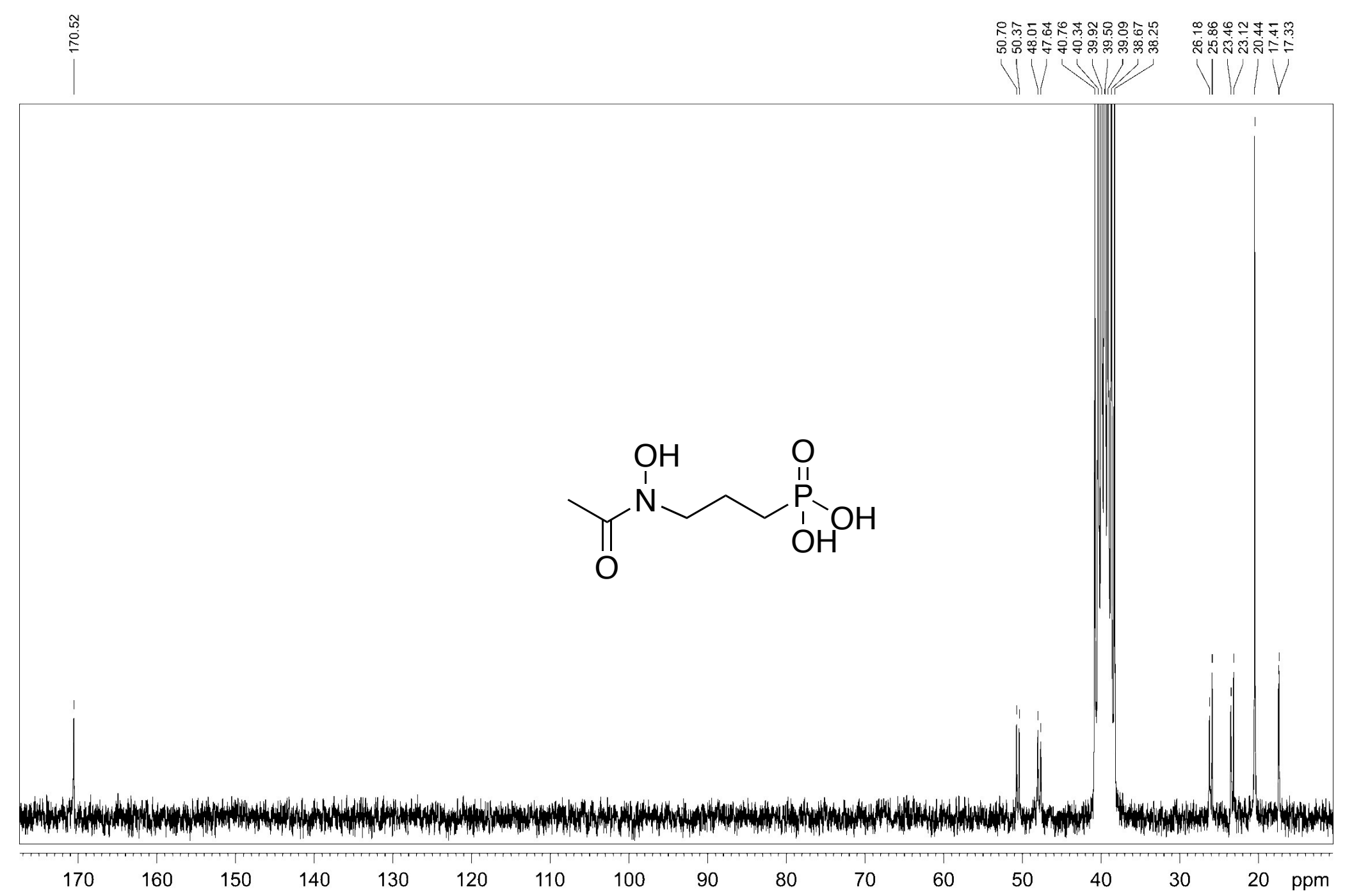

Figure S12. The ${ }^{13} \mathrm{C}$ NMR spectra of the compound $\mathbf{1 3}$. 


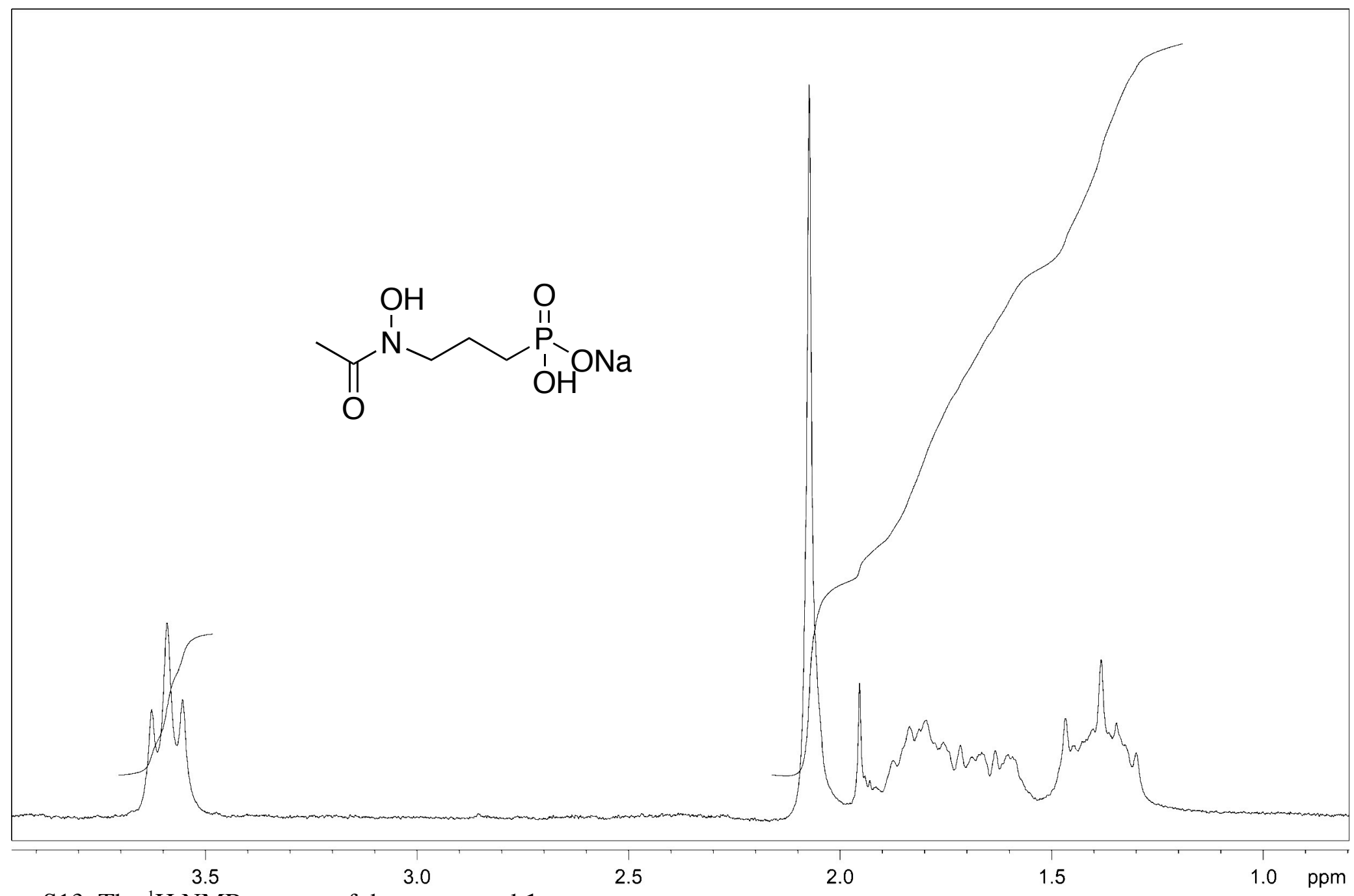

Figure S13. The ${ }^{1} \mathrm{H}$ NMR spectra of the compound $\mathbf{1}$. 


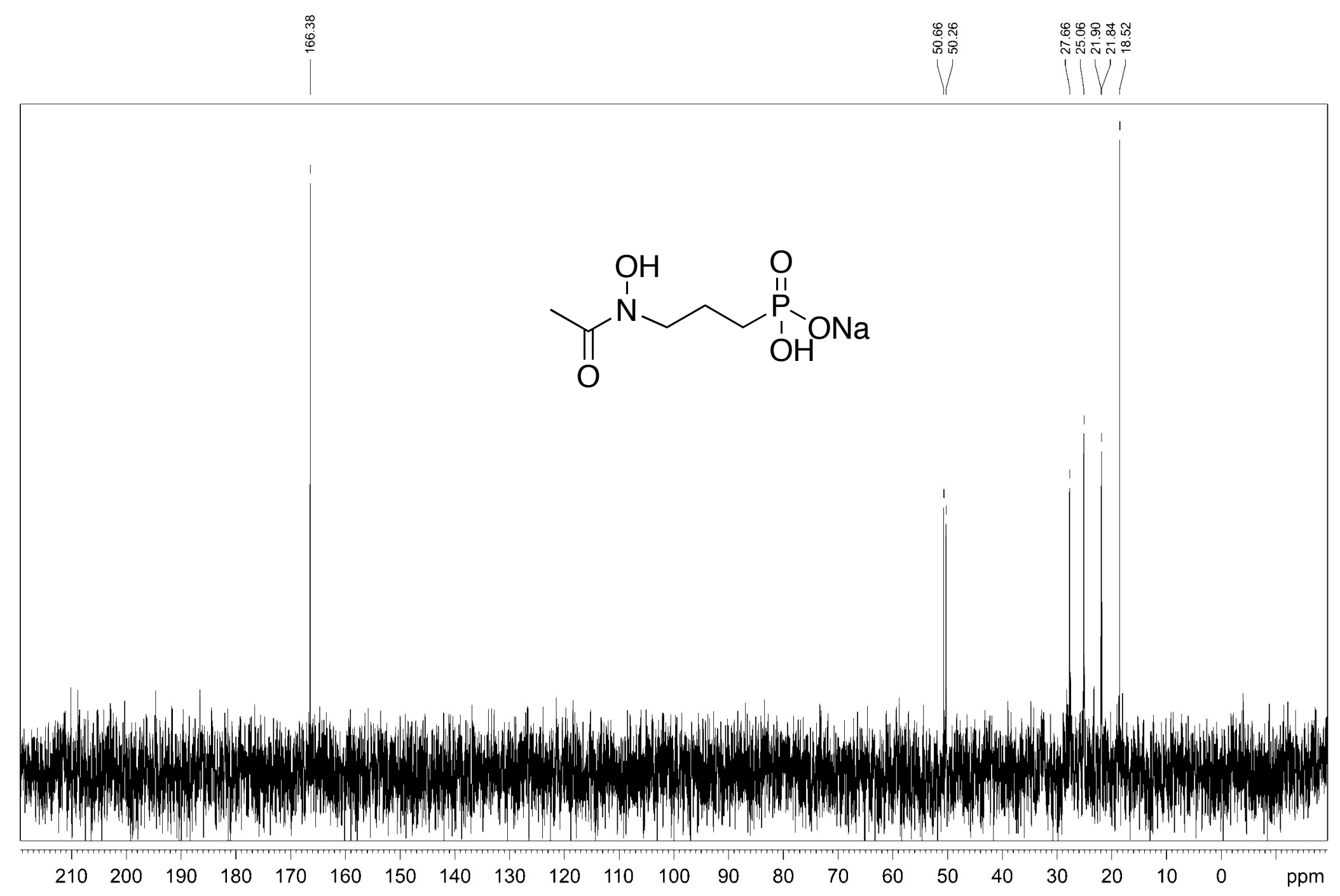

Figure S14. The ${ }^{13} \mathrm{C}$ NMR spectra of the compound $\mathbf{1}$. 